\title{
Descripción del nido y cuidado parental de Euphonia chlorotica (Aves: Fringillidae) en Santa Cruz, Bolivia
}

\author{
Description of the Nest and Parental Care of Euphonia chlorotica (Birds: \\ Fringillidae) in Santa Cruz, Bolivia
}

\author{
Efraín Miguel Peñaranda Barrios \\ Geología y Recursos Naturales - GeoAmbiente Ltda., Casilla 1569. Santa Cruz-Bolivia. \\ * Autor corresponsal: epenaranda@cotas.com.bo
}

\section{RESUMEN}

En este estudio se describen los primeros datos sobre aspectos reproductivos de Euphonia chlorotica en Bolivia mediante el monitoreo de un nido construido en un macetero colgante dentro una vivienda ubicada en la ciudad de Santa Cruz de la Sierra. El nido tenía forma globular con entrada lateral típica de la especie y fue construido en un lapso de 6 días en diciembre de 2019. Tres huevos de color blanquecino con manchas café oscuras fueron incubados durante 16 días exclusivamente por la hembra. Los pichones nacieron de forma sincrónica el 25 de enero de 2020 y luego de 16 días abandonaron el nido con sus plumajes totalmente desarrollados, excepto por el largo de las plumas de la cola, y con los picos aun con la comisura amarilla característica de los juveniles. La alimentación de los pichones fue realizada por ambos progenitores y la probabilidad de supervivencia diaria del nido y éxito de anidación fue del 100\%, pese a encontrase en un ambiente altamente urbanizado.

Palabras clave - Biología reproductiva, cuidado biparental, tamaño de puesta, incubación, ambiente urbano.

\section{ABSTRACT}

This study describes the first data on reproductive aspects of Euphonia chlorotica in Bolivia by monitoring a nest built in a hanging planter inside a house located in

Ref. bibliográfica: Peñaranda Barrios, E. M. 2020. “Descripción del nido y cuidado parental de Euphonia chlorotica (Aves: Fringillidae) en Santa Cruz, Bolivia". Acta zoológica lilloana 64 (2): 166-175. Fundación Miguel Lillo, Tucumán, Argentina. D.O.I.: https://doi.org/10.30550/j.azl/2020.64.2/6

> Recibido: 13 de mayo 2020 - Aceptado: 26 de agosto 2020.

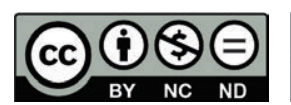

> URL de la revista: http://actazoologica.lillo.org.ar

- Esta obra está bajo una Licencia Creative Commons Atribución - No Comercial - Sin Obra Derivada 4.0 Internacional. 
Santa Cruz de la Sierra. The nest was globular in shape with a lateral entrance typical of the species and was built within 6 days in December 2019. Three whitish eggs with dark brown spots were incubated for 16 days exclusively by the female. The nestlings hatched synchronously on January 25, 2020 and after 16 days they left the nest with their plumage fully developed, except for the length of the tail feathers, and with the beaks still with the characteristic yellow commissure of the juvenile. The feeding of the nestlings was carried out by both parents and the probability of daily nest survival and nesting success was 100\% despite being in a highly urbanized environment.

Keywords - Reproductive biology, biparental care, clutch size, incubation, urban environment.

Euphonia chlorotica (Doradito o Tangará común), es una especie de ave paseriforme de la familia Fringillidae ampliamente distribuida en el norte y centro de América del Sur, llegando hasta el norte de Argentina y Uruguay (Hilty, 2011; eBird, 2020). En Bolivia se la encuentra en los departamentos de Pando, Beni y Santa Cruz, norte de los departamentos de Cochabamba y La Paz, y este de los departamentos de Chuquisaca y Tarija (Remsen y Traylor, 1989; Herzog et al., 2017). Habita principalmente bosques y arbustos secos a semihúmedos, bordes de bosque húmedo a hiperhúmedo, vegetación del Cerrado, humedales, vegetación secundaria, pastizales, pueblos, jardines y parques urbanos de tierras bajas y ladera este de los Andes (Ridgely y Tudor, 1994; Herzog et al., 2017; BirdLife International, 2018). En la mayor parte de su distribución se la encuentra hasta los 2000 msnm y en Bolivia es común encontrarla hasta los $2600 \mathrm{msnm}$ (Herzog et al., 2017); excepcionalmente, su rango altitudinal puede llegar hasta los $3300 \mathrm{msnm}$ (Balderrama, 2006). Al igual que otras especies del género Euphonia, se caracteriza por tener una dieta frugívora altamente especializada y por la construcción de nidos globulares con una entrada lateral (Sick, 1997; Isler e Isler, 1999; Hilty, 2011).

A pesar de ser una especie frecuente en su rango de distribución, el conocimiento de la biología reproductiva de $E$. chlorotica ha sido escasamente reportado. Datos sobre la biología reproductiva de la especie provienen de observaciones y descripciones en Paraguay (Bertoni, 1904), norte de la Argentina (de la Peña, 1996 y 2013; Di Giacomo, 2005), sur y sureste del Brasil (Mauricio et al., 2013; Perrella et al., 2017).

En este trabajo se describen por primera vez aspectos básicos de su reproducción en Bolivia, incluyendo tamaño de puesta y comportamientos de incubación en un ambiente urbano de la ciudad de Santa Cruz de la Sierra.

Las observaciones se realizaron en un ambiente altamente urbanizado de la ciudad de Santa Cruz de la Sierra, departamento Santa Cruz, Bolivia (17 $47^{\prime} 48^{\prime \prime}$, $63^{\circ} 05^{\prime} 54.4^{\prime \prime} \mathrm{O}, 400 \mathrm{msnm}$ ). La zona se caracteriza por tener viviendas con jardines interiores, delimitadas por calles y avenidas asfaltadas con aceras y jardineras donde se desarrollan arbustos y árboles de los géneros Handroanthus, Tabebuia, Erythrina, Terminalia y Persea, intercalados con palmeras de la familia Arecaceae (ej.: Cocos 
nucifera, Attalea Phalerata) e higueras de la familia Moraceae (Ficus sp.). El clima es cálido y húmedo, con una temperatura media anual que varía entre 24 y $31^{\circ} \mathrm{C}$, y una precipitación promedio anual de $1321 \mathrm{~mm}$ (PDTI, 2019).

Entre el 7 de diciembre de 2019 y el 11 de febrero de 2020 se realizaron observaciones de un nido semiexpuesto construido de manera singular. El 7 de diciembre de 2019, se registró por primera vez a la pareja de E.chlorotica construyendo el nido en una maceta colgante plástica de $28 \mathrm{~cm}$ de diámetro y $19 \mathrm{~cm}$ de altura, en el jardín interior de una vivienda. La maceta colgaba a $220 \mathrm{~cm}$ del suelo y se encontraba 30 $\mathrm{cm}$ por debajo de un alero de $90 \mathrm{~cm}$ de ancho. La maceta contenía un helecho del género Nephrolepis y estaba expuesta al sol el 100\% de su superficie entre las 10:00 h y las 17:00 h (Figura 1A-B). La vivienda tenía incorporadas en su alero, una hilera de macetas de iguales características al sitio de nidificación y se encontraban separadas entre sí cada $120 \mathrm{~cm}$, conteniendo helechos de los géneros Aspleniun, Nephrolepis y Dryopteris, y plantas angiospermas como Asparagus, Anthurium y Monstera. Tanto el macho como la hembra construyeron el nido, a veces vocalizando previo ingreso. Cada visita durante la construcción duraba entre 1 y 3 min para traer el material y acomodarlo. En 7 horas de observación (los días 2 y 4), el macho visitó el nido 135 veces, la hembra solo 96 . La hora de mayor actividad fue entre las $06: 00 \mathrm{~h}$ y 08:30 h, donde el macho en el día 2 visitó el nido 19 veces y la hembra 15 veces. Los recesos entre visitas al nido eran de hasta 2 horas. El material era obtenido principalmente de las plantas disponibles en el interior del jardín; como aglomerante las aves utilizaron telas de araña (Figura 1C). Cuando la hembra acomodaba el nido, el macho perchaba en un cable eléctrico a unos $\sim 5.5 \mathrm{~m}$, haciendo una vocalización de llamada doble al llegar o al irse del sitio. Entre el día 4 a 6, se observó que la hembra pasaba más tiempo en el nido y realizaba pequeñas vibraciones con su cuerpo posiblemente para acomodar la cámara de incubación. En total se emplearon 6 días en la construcción del nido. Al finalizar, la pareja se alejó de la vivienda y retornaron nuevamente el 6 de enero de 2020.

El nido era de estructura semiesférica, globular pero achatado lateralmente para mejorar su adhesión al sustrato de la maceta, y a las hojas y tallos de la planta hospedera. La entrada lateral y ligeramente superior estaba bien definida y delimitada por fibras flexibles colocadas a su alrededor, su forma era redonda al inicio de la construcción y terminó elíptica en las últimas etapas de desarrollo de las crías, a esto coadyuvó la marchitez de la planta hospedera por falta de riego que prácticamente deformó el nido (Figura 1D, 2B y 2C). El nido estaba revestido externamente con fibras vegetales, raquis de helechos, ramas delgadas, hojas secas, plumones e hilos de algodón, todo compactado con tela de araña. La cámara de incubación estaba recubierta por musgos, fibrillas de helechos, plumas, cabellos y cordones de nylon. Las medidas del nido fueron: diámetro externo $8.5 \mathrm{~cm}$; diámetro interno $5.2 \mathrm{~cm}$; altura externa $9,5 \mathrm{~cm}$ y altura interna $6,8 \mathrm{~cm}$. El grosor de las paredes en promedio fue de $2 \mathrm{~cm}$ dada la estructura y forma del nido. La entrada del nido tuvo un diámetro de $4.4 \mathrm{~cm}$ y orientación hacia el oeste (Figura 1B).

La puesta inicio el 7 de enero de 2020 y concluyó dos días después, poniendo en total 3 huevos, uno cada día de manera consecutiva. Los huevos fueron ovoidales de color blanquecino, con manchas castaño oscuro en toda su superficie, pero más 

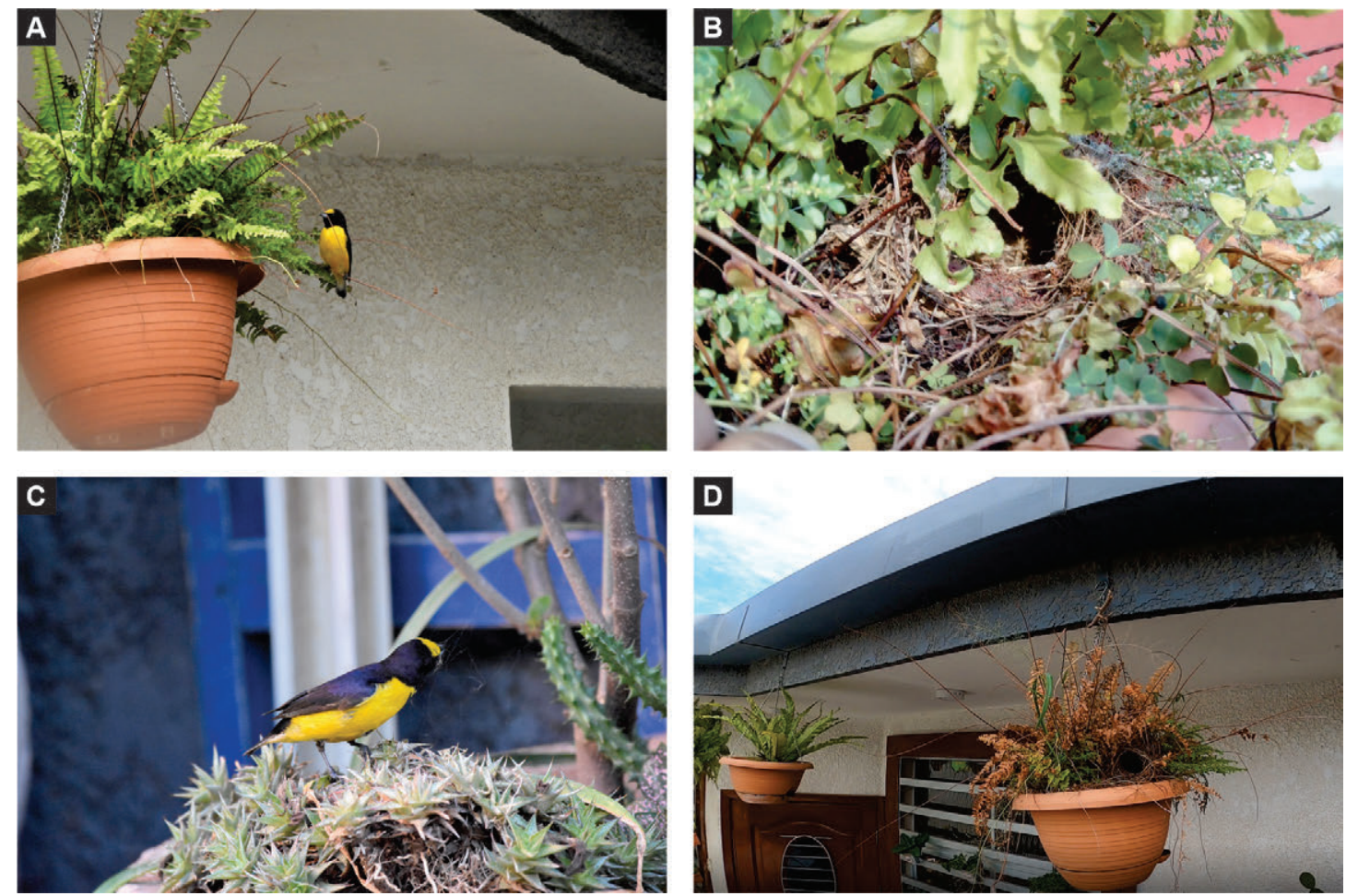

Figura 1. Construcción del nido de Euphonia chlorotica en un ambiente urbano de la ciudad de Santa Cruz de la Sierra (Fotos A-D: Kendra Peñaranda). (A) Llegada del macho a la planta soporte para el nido. (B) Vista frontal del sitio de ubicación del macetero colgante donde se ubicó el nido y distancia al acceso principal. (C) Obtención de telas de araña en plantas del jardín interior para la construcción del nido. (D) Nido semiexpueto, estructura globular y con entrada lateral de orientación oeste.

Figure 1. Construction of the nest of Euphonia chlorotica in an urban environment in the city of Santa Cruz de la Sierra (Photos A-D: Kendra Peñaranda). (A) Arrival of the male at the support plant for the nest. (B) Front view of the location of the hanging planter where the nest was located and distance to the main access. (C) Obtaining spider webs on indoor garden plants for nest construction. (D) Semi-exposed nest, globular structure and with a west-facing side entrance.

densas y más grandes en su polo mayor. Sus medidas fueron: longitud 15.7 a 16.2 $\mathrm{mm}$; ancho 12 a $12.1 \mathrm{~mm}$ y peso 1.1 a $1.3 \mathrm{~g}$ (Figura $2 \mathrm{~A}$ ).

El periodo de incubación tuvo una duración de 16 días luego de la puesta del último huevo y estuvo a cargo exclusivamente de la hembra (Figura 2B). El macho fue visto esporádicamente entre el mediodía o el crepúsculo, generalmente perchado o realizando llamados de contacto con la hembra, la cual respondía desde el nido. El macho no alimentaba a la hembra durante la incubación, sino que ella misma salía a buscar su alimento. La Duración promedio de los intervalos de incubación fue de $21.7 \pm 27 \mathrm{~min}$ (rango 5-32 min). Las interrupciones entre intervalos de incubación fueron variables y asociadas a la alimentación o actividad de personas en la vivienda que en ocasiones alteraba a la hembra, la cual salía volando emitiendo un canto agudo. Durante la noche la incubación de parte de la hembra fue continua.

La eclosión de los huevos se evidenció por las visitas que comenzó a hacer el macho al nido con alimento. Los pichones nacieron de forma sincrónica el 25 de enero, con un plumón gris en la región dorsal, cefálica, lumbar y humeral; los ojos cerrados y la piel era de color gris oscuro, el pico era negro con las comisuras ama- 

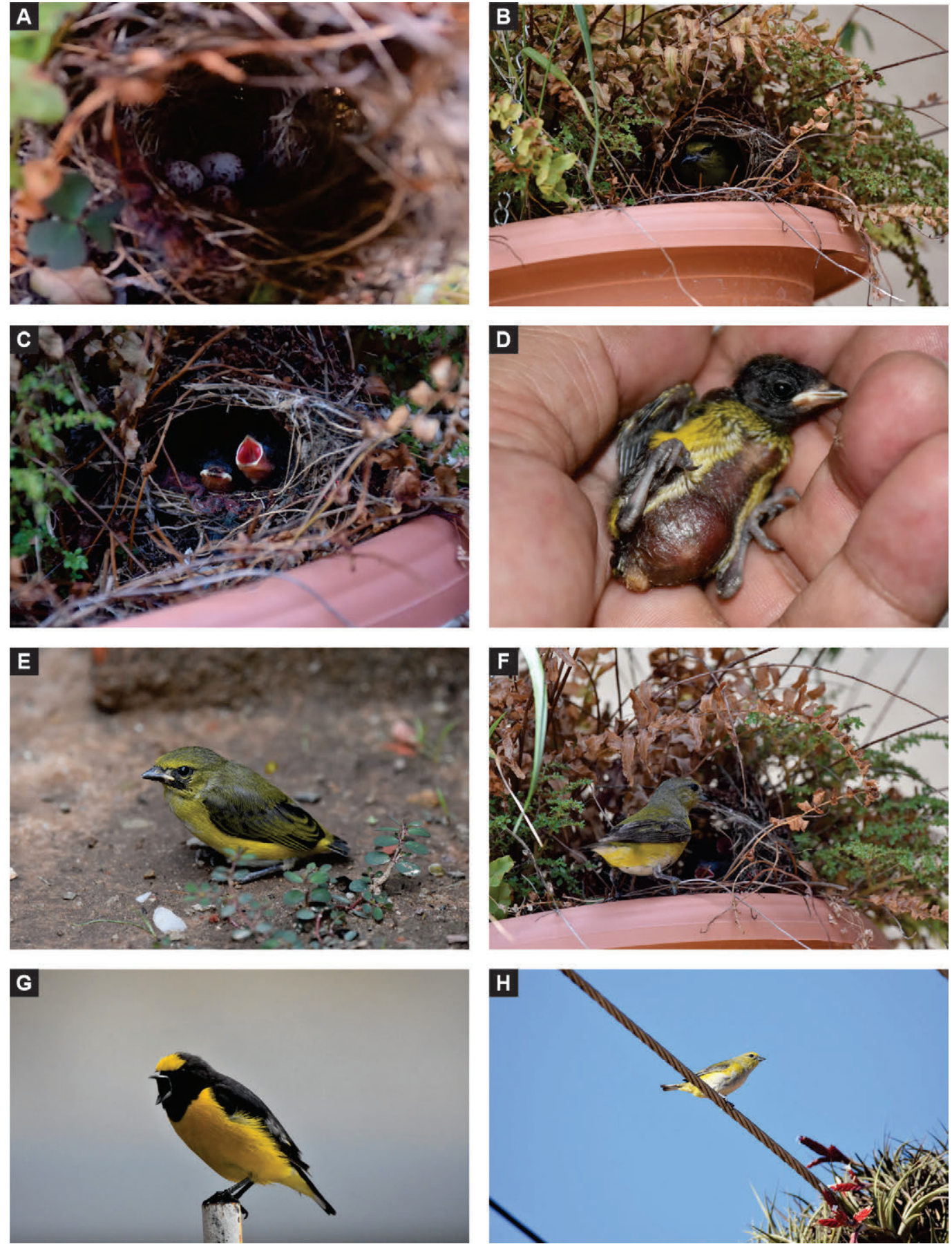

Figura 2. Puesta, desarrollo de los polluelos y cuidados parentales de Euphonia chlorotica (Fotos A-H: Efraín Peñaranda). (A) Vista general del nido, taza profunda y huevos. (B) Hembra en periodo de incubación. (C) Polluelos día 4. (D) Día 7. (E) Juvenil al salir del nido en el día 15 (volantón). (F) Hembra alimentado a los polluelos. (G) Macho vocalizando en una percha a $1 \mathrm{~m}$ del nido. (H) Hembra de $E$. chlorotica construyendo un nido en un clavel del aire (Tillandsia didisticha) desarrollado sobre un cable eléctrico. El nido se observó el 24/10/2016 y se situaba a $10 \mathrm{~km}$ de distancia del nido monitoreado en el presente estudio.

Figure 2. Laying, development of chicks and parental care of Euphonia chlorotica (Photos A-H: Efraín Peñaranda). (A) General view of the nest, deep cup and eggs. (B) Female in incubation period. (C) Chicks day 4. (D) Day 7. (E) Juvenile when leaving nest on day 15. (F) Female fed to the chicks. (G) Male vocalizing on a perch $1 \mathrm{~m}$ from the nest. $(\mathrm{H})$ E. chlorotica female building a nest in Tillandsia didisticha developed on an electric cable. The nest was observed on 10/24/2016 and was located $10 \mathrm{~km}$ away from the nest monitored in the present study. 
rillas y el paladar rosáceo intenso (Figura 2C). En el sexto día, todos los pichones abrieron los ojos, presentaban plumones de color amarillo verdoso en el dorso, en las barbas de los cálamos de las remeras primarias y secundarias y en los plumones de la frente. En los flancos, se observaron plumas más desarrolladas de color amarillo intenso (Figura 2D). En el día 14 casi todo el plumaje de los polluelos estaba completo y ocupaban el $100 \%$ de la cavidad del nido. En esta etapa, las plumas de las colas se encontraban en un estado intermedio de desarrollo y los picos aún tenían las comisuras amarillas (Figura 2E). El 10 de febrero, luego de 16 días de desarro1lo, los polluelos abandonaron el nido, inicialmente hicieron un vuelo corto hasta el suelo, y luego con pequeños saltos y un vuelo errático se posaron en un lapacho rosado (Handroanthus impetiginosus). Durante los tres días posteriores al abandono del nido se los escuchó piar en el lapacho; eran alimentados por ambos padres y luego desaparecieron. El 20 de febrero se observó por última vez a los padres acercarse al nido y luego retirarse.

Tanto el macho como la hembra se ocuparon de la alimentación de los polluelos. La primera provisión de comida se dio entre las 6:00 y 6:30 h; mientras que la última, fue entre las $18: 30$ a 18:45 h. Durante los primeros 10 días la hembra intercalaba el aprovisionamiento de comida con el empollamiento de pichones, por las noches permanecía en el nido cuidando sus polluelos. Las cabezas de las crías fueron vistas a partir del día 3 mientras pedían comida, y reaccionaban ante cualquier movimiento cerca del nido. Luego del día 10 días, las crías fueron alimentadas desde fuera del nido y ninguno de los progenitores permanecía con ellos durante la noche. La alimentación en general iniciaba con la llegada de los progenitores hasta una percha próxima al nido, el macho emitía regularmente un canto trisílabo pausado fiu...fiu...fiuuuu...cada 4 a $6 \mathrm{~s}$; posteriormente macho y hembra se acercaban a una distancia de 1 a $2 \mathrm{~m}$ del nido y emitían pitidos suaves o vocalizaciones que eran respondidos por los polluelos (Figura 2F-G). Cuando solo uno de los progenitores llegaba cerca al nido, este emitía cantos bisílabos y a los pocos segundos aparecía la pareja. En general, el macho alimentaba primero a las crías, mientras la madre permanecía vigilando, y luego se invertían los roles. Ante un elemento nuevo e intrusivo cerca al nido, el macho realizó comportamientos agonísticos: como "halconeos" muy próximos al objeto, brincos, aleteos y balanceos de cabeza y cuerpo; luego de verificar que el objeto no representaba riesgo, se posaba nuevamente cerca al nido. El tiempo de alimentación de las crías por parte de los padres fue comparado entre el día 7 y 12. El macho empleo un tiempo de $61 \pm 88$ s (rango 7-95 s) para alimentar a los polluelos; mientras que de la hembra, 50,2 \pm 73 s (rango 4-77 s). La prueba de $t$ efectuada no mostró diferencias estadísticamente significativas en las visitas al nido con alimento entre el macho y la hembra durante los días evaluados $(\mathrm{t}=1,51 ; \mathrm{p}=0,139, \mathrm{gl}=38) . \mathrm{El}$ intervalo entre visita y visita para alimentar a los polluelos fue de $60 \pm 116 \mathrm{~min}$. No se pudo determinar si lo volantones continuaron siendo alimentados por los padres luego de abandonar el nido, o si llegaron a una fase adulta o fueron depredados.

Se registraron valores de ruido en la vivienda de entre 45 y $91 \mathrm{~dB}(\mathrm{~A})$. Los ruidos más fuertes generados fueron por herramientas de corte, los cuales generalmente eran de corta duración y alta intensidad. Otros ruidos menores eran provocados por 
los trabajadores así como el ruido de fondo provocando por el tráfico vehicular. $E$. chlorotica en respuesta a estas perturbaciones no mostró ningún efecto en cuanto a la construcción del nido, incubación o éxito reproductivo; excepto, cuando las distancias de los trabajadores eran menores a $2 \mathrm{~m}$ del nido o de alguno de los progenitores, en este caso se retiraban del sitio a una percha próxima emitiendo un silbido agudo y permanecían vigilando para luego retomar sus actividades.

El presente trabajo presenta la primera descripción del nido, huevos y pichones, así como el comportamiento reproductivo de E. chlorotica en Bolivia. La temporada de anidación coincidió con los registros de nidos activos citados por Lima (2006) y Perrella et al. (2017) para Brasil, pero difieren de los resultados mostrados por Bertoni (1904) para Paraguay, de la Peña (1996) y Di Giacomo (2005) para Argentina, Mauricio et al. (2013) para el sur de Brasil y Arango (2016) para Colombia. Concordamos con Perrella et al. (2017) en que la época de reproducción puede variar según la zona biogeográfica de América del Sur; y en Santa Cruz de la Sierra probablemente pueda extenderse de septiembre a enero, por observaciones de nidos realizadas por el autor en localidades próximas al sitio de nidificación (Figura $2 \mathrm{H}$ ).

Las características constructivas del nido, tamaño de la nidada, color y forma de los huevos fueron similares a lo descrito por Di Giacomo (2005), Lima (2006) y Perrella et al. (2017). El tiempo de construcción del nido fue menor al señalado por de la Peña (1999 y 2013) posiblemente por las condiciones particulares de su ubicación y las adaptaciones sinantrópicas de la especie. El empleo de telas de araña como aglomerante coincide con lo señalado por Skutch (1954) para el género Euphonia y por Perrella et al. (2017) para E. chlorotica (Figura 1C). En los materiales de construcción se observa por primera vez el empleo de cabellos, hilos de algodón y material plástico (Figura 2C). Al respecto McKinney (2002), menciona que, en comparación con los hábitats rurales, los nidos de los entornos urbanos se caracterizan por el aumento de material antropogénico y reducción del material natural.

El periodo de incubación fue de 16 días, mayor al presentado por Di Giacomo (2005), Arango (2016) y Perrella et al. (2017). Di Sallo, Bodrati y Cockle (2019), señalan diferentes periodos de incubación para el género Euphonia: 14 a 16 días para E. hirundinacea, 18 para $E$. imitans, 17 para E. minuta, 13 a 16 para E. laniirostris, 16 a 17 para E. pectoralis y 14 para E. chlorotica. Según Di Sallo et al. (2019), el período varía considerablemente entre las diferentes especies de Euphonia, posiblemente debido a factores ecológicos, que podrían ser dilucidados en estudios comparativos que involucren más nidos y especies. La protección del nido por parte de los padres difiere entre las etapas de anidación. La hembra es la encargada de la incubación de los huevos y el macho ocasionalmente de la protección o vigía (aunque este no se acerca al nido durante la incubación), lo que supone un mayor esfuerzo de la hembra en esta etapa de la biología reproductiva, resultados similares se encuentra descritos en Arango (2016) y Perrella et al. (2017). Adicionalmente, el comportamiento de cuidado biparental de los polluelos observado en el presente trabajo, es ampliamente descrito para las Euphonias y otras aves como una estrategia para incrementar descendencia y lograr futuros reproductores (Drent y Daan, 1980; Clutton-Brock, 1991; Carranza, 2002). 
El sitio elegido para la construcción del nido así como la planta soporte es un caso singular para la nidificación de la especie. No se conocen cuáles son los rasgos relevantes para la selección del sitio de nidificación por E. chlorotica, pero se entiende que este es uno de los factores determinantes para el éxito de su reproducción (Collias, 1997).

Se deberá continuar estudiando la biología reproductiva de estas y otras especies en ambientes urbanos para determinar qué patrones poblacionales se están modificando (ej., densidad poblacional, supervivencia de juveniles y adultos, etc.) o conocer cómo la avifauna se ve afectada o se sustenta en estos ambientes.

\section{AGRADECIMIENTOS}

A la empresa GeoAmbiente Ltda., por el apoyo con el material de campo, vehículos y equipos fotográficos. A José y Carlos Peñaranda B., Irene Estremadoiro y Kendra Peñaranda E., por el apoyo incondicional en las observaciones y fotografías de campo. A Ivan Linneo por el apoyo en la identificación de las especies de plantas ornamentales. Agradecemos también a los revisores anónimos por sus recomendaciones para mejorar el manuscrito.

\section{FINANCIAMIENTO}

Este trabajo fue posible gracias al financiamiento de GeoAmbiente Ltda.

\section{PARTICIPACIÓN}

Efraín Miguel Peñaranda Barrios: muestreo de campo, análisis de datos y redacción del artículo.

\section{CONFLICTOS DE INTERÉS}

No existen conflictos de interés entre los autores ni con terceros.

\section{LITERATURA CITADA}

Arango, C. (2016). Eufonia gorgipúrpura (Euphonia chlorotica). Wiki Aves de Colombia. Universidad Icesi. Cali Colombia. https/www.icesi.edu.co.

Balderrama, J. A. 2006. Diversidad, endemismo y conservación de la ornitofauna del Parque Nacional Tunari (Cochabamba, Bolivia). Ecología en Bolivia 41(2):149170. 
Bertoni, A.W. (1904). Contribución para el conocimiento de las aves del Paraguay. Anales Científicos Paraguayos 3:1-10.

BirdLife International. (2018). Euphonia chlorotica. La Lista Roja de Especies Amenazadas de la UICN 2018: e.T22722706A132017181. https://dx.doi.org/10.2305/ IUCN.UK.2018-2.RLTS.T22722706A132017181.en. Descargado el 28 de abril de 2020.

Carranza, J. (2002). La evolución del cuidado parental. En: Evolución, la base de la biología (ed. Soler, M.). 193-212. Proyecto Sur Ediciones. España

Clutton-Brock, T.H. (1991) The evolution of parental care. Princeton University Press. Princeton (Estados Unidos).

Collias, N. (1997). On the origin and evolution of nest building in passerine birds. Condor 99:253-270.

De la Peña, M.R. (1996). Descripción de nidos nuevos o poco conocidos de la avifauna Argentina. El Hornero 14: 085-086.

De la Peña. M.R. (2013). Nidos y reproducción de las aves argentinas. Ediciones Biológicas. Serie Naturaleza, Conservación y Sociedad N 8. Santa Fe, Argentina.

Di Giacomo, A. G. (2005). Aves de la Reserva El Bagual (201-465). En Di Giácomo, A. G., \& S. Krapovickas (eds.). Historia natural y paisaje de la Reserva El Bagual, Provincia de Formosa. Temas de Naturaleza y Conservación, Monografía de Aves Argentinas No 4, Buenos Aires, Argentina.

Di Sallo, F.G., A. Bodrati, K. L. Cockle. (2019). Nesting and natural history of the chestnut-bellied euphonia (Euphonia pectoralis) in Misiones, Argentina, and comparison with other species in the genus. Ornitología Neotropical. 30:19-26.

Drent, R.H., Daan, S. (1980). The prudent parent: energetic adjustments in avian breeding. Ardea 68, 225-253.

eBird. 2020. The Cornell Lab. of ornithology. Mapa de distribución de Euphonia chlorotica. Acceso en: https://ebird.org/map/puteupl?neg=true\&env.minX=\&env. $\min \mathrm{Y}=$ \&env. $\max \mathrm{X}=$ \&env.max $\mathrm{Y}=\& \mathrm{zh}=$ false $\& \mathrm{gp}=$ false \&ev $=\mathrm{Z} \& \mathrm{mr}=1-12 \& \mathrm{~b}$ $\mathrm{mo}=1 \& \mathrm{emo}=12 \& \mathrm{yr}=\mathrm{all} \& \mathrm{byr}=1900 \& \mathrm{eyr}=2020$.

Herzog, S.K., Terrill, R.S., Jahn, A.E., Remsen Jr, J.V., Maillard, O., García-Solíz, V.H., Macleod, R., Maccormick, A. y Vidoz, J.Q. (2017). Birds of Bolivia. Field Guide. Asociación Armonía. Santa Cruz.

Hilty, S. (2011). Family Thraupidae (Tanagers). En: Del Hoyo J., Elliot A., Christie D.A. (Eds). Handbook of the Birds of the World: Tanagers to New World Blackbirds. Lynx Edicions, Barcelona, 46-329.

Isler, M.L., Isler P.R., (1999) The Tanagers: natural history, distribution and identification. Smithsonian Institution, Washington, DC.

Lima, P.C. (2006). Euphonia chlorotica chlorotica (Linnaeus, 1766), um ninho diferente: adaptação ou evolução? Atualidades Ornitológicas 129: 8-9.

Mauricio, G.N., G. Bencke, M. Repenning, D. Borges, R. Dias, L. Bugoni. (2013). Review of the breeding status of birds in Rio Grande do Sul, Brazil. Iheringia, Série Zoologia, Porto Alegre, 103(2):163-184.

McKinney M. L. (2002). Urbanization, biodiversity, and conservation. BioScience, 52: 883-890. 
PDTI. (2019). Plan Territorial de Desarrollo Integral Santa Cruz de la Sierra 20162020. GAM-SCZ.

Perrella, D.F, Davanço, P.V., Oliveira, L.S., Sousa, L., Mercival, R.F. (2017). Reproductive aspects of the Purple-throated Euphonia, Euphonia chlorotica (Aves: Fringillidae) in southeastern Brazil, and first record of the species nesting inside a vespiary. Zoologia 34:15.

Remsen, J.V., Traylor, M.A. (1989). An Annotated List of the Birds of Bolivia. Buteo Books, Vermillion, South Dakota 79 pp.

Ridgely, R. S., Tudor, G. (1994). Birds of South America. Volume II. The Suboscine passerines. University of Texas Press, Austin, USA.

Sick, H, (1997). Ornitologia brasileira: Uma Introducao. Ed. Nova Fronteira, Rio de Janeiro.

Skutch, A.F. (1954). Life histories of Central American birds: families Fringillidae, Thraupidae, Icteridae, Parulidae and Coerebidae (No.598.29728 S629). Cooper Ornithological Society, Berkeley, California, USA. 